\title{
Analysis on Negative Transfer of Native Language Syntax Structure in English Compositions of Chinese College Students
}

\author{
Xiaoqing Sun \\ College of Fundamental Education, Shanghai University of Engineering Science, Shanghai, 201620, \\ China
}

Keywords: Syntax structure. Parataxis. Hypotaxis. Influence

\begin{abstract}
The differences of English and Chinese in syntax structure are analyzed and the influence of these differences on the Chinese English learners and the method to solve the negative impact is explored.
\end{abstract}

\section{Introduction}

Whether the learners have realized, the influence of transfer in foreign language learning is undisputed. Transfer means the influence caused by the commonness and difference between the target language and any other acquired (or not completely acquired) language. Odlin (1989). The transfer of native language includes positive transfer and negative transfer: positive transfer plays a promoting role for the second language acquisition process, while the negative transfer obstructs the acquisition. The more similar between the native language and second language is, the easier it is to generate positive transfer, on the contrary, it is easier to generative transfer. Ellis (1994) once pointed out: in the students with German as the native language investigated by Grauberg in 1971, it was found that the negative transfer caused by native language accounted for $36 \%$ of the language error; through research, Chau found that the cause caused by the negative pragmatic transfer of the Chinese accounted for $51 \%$ of the language error. Mukkatash(1977) indicated through research that the error of negative pragmatic transfer caused by the native language in the Arabians accounted for $23 \%$ of the language error. When researching the Spanish in 1980, Flink found that the error of negative pragmatic transfer accounted for 31\% of the language error. Most of the contemporary Chinese college students began to learn English in junior middle school when they had mastered their native language quite skillfully. In the process of learning English, as the original experience, the native language is a preparation in cognition for the learning of a new language, which will inevitably participate in the language of a new language. In syntax level, English and Chinese have the following differences:

\section{Chinese is of parataxis, terse but forceful; English is of hypotaxis with a clear structure}

Hypotaxis means that the subordinated structure in a sentence has the associated words representing the sentence relation, and the grammar forms and means expressing the syntax relation. Parataxis means that the sentences are connected without associate words representing the syntax relation and without typical grammar forms and means. Both hypotaxis and parataxis will be used in English and Chinese, but there is a large different of proportion and frequency. English stresses on hypotaxis, while Chinese stresses on parataxis (Zhou Jianbin, 2003).

The Chinese sentence presents a bamboo pole structure, with the mutual combination of components most depending on the penetration of semantics, reflecting of context and arrangement of word sequence, and usually there are no connection words used and the logistic relation is represented by the word order. The Chinese is in time sequence through the collocation of many verbs or "run-on sentence, with things stated clearly one by one, presenting a run-on pattern in time sequence. The grammar of Chinese is implicit, and even if the logic relation between sentences is not connected with conjunction, the readers can understand the meanings through the logic relation hidden in the language, while English is a hypotaxis language, with a tree-type sentence structure, 
including trunk and branches. The sentences set up other components layer by layer through a lot of infinitives, participles, prepositions, conjunctions, relation pronouns and relation adverbs reflecting the form relationship with the S-V structure as the trunk and verb as the center, presenting a spatial pattern of spreading from the center to outside, with rigid and compacted structure. For example, in the sentence We should take a firm hold of the overall interests of sino-US relations and settle our differences properly so as to reach the goal of promoting mutual understanding, broadening common ground, developing cooperation and building a future together. There are conjunctions and adverbs added in English. During writing, students will be influenced by the Chinese habit, and connect several simple sentences directly with comma without any conjunction. When connected with sentences, many simple sentences present a linear structure, for example, Life in the city is much more expensive than in the countries, the crime rate in the city is several times higher than in the countryside. Another example, I was born in a village, I studied there for 9 years, I love there very much, such sentences connected by coma are influenced by the run-on sentence of the Chinese. The Chinese sentence is limited to the parataxis, with a large flexibility in the information contents of sentences due to no limitation in grammar form. The sentence is connected one by one and can be expanded infinitely. But English is a hypotaxis language, constructing sentences with subject and predicate as the center. If it is required to express more meanings in a sentence, the meanings should be expressed by conjunctions and relation pronouns plus the subordinated clause.

\section{The Chinese sentence pattern highlights the topic; English sentence pattern highlights the subject}

The Chinese is a language paying attention to the topic, with the basic sentence structure of information unit topics and the language commented. Mr. Zhao Yuanren once pointed out that 50\% of the Chinese sentence structures belong to "topic-description" frame. The talker can select different sentence expression methods according to the communication effect to achieve, especially talk about the information for the purpose of emphasizing a piece of primary and new information, and then make a supplementary description to this information. The topic is target that the talker want to express. The main part of the sentence is the component of the topic described, which describes, explains and challenges the topic. It is very common in the Chinese sentence that the topic coincides with the subject or that there is no subject. And the basic sentence structure is centered on the topic or subject. For example, in class, no arbitral speaking is allowed. The bold parts are the subjects, and the others are for description or comment. English is a language emphasizing the form logistics, emphasizing the subject and the basic sentence structure is centered on $\mathrm{S}+\mathrm{V}$, for example, "It is necessary to make people become aware of the importance of protecting environment.”

\section{More passive voices are used in English than in Chinese}

In terms of the division of active sentences and passive sentences, "it is mainly to review the condition of subject. Subject is the actor of predicate action, i.e. the sentence in which the subject is the actor is called sensitive sentence, and the sentence in which the subject is the carrier of predicate action, i.e. the subject is the acceptor is called passive sentence. (Liu Lanying, 2001).

The passive forms of Chinese is often expressed by many prepositions, without change in syntax or verb form. These prepositions introduce the actor, and meanwhile indicate that the subject in the sentence is the object of action. Unlike Chinese, the passive voice in English is often expressed by the auxiliary be which expresses the passive voice and the past participle of the main verb, which both needs the change of morphology and the change of syntax. The passive sentences are widely used in English, and the use frequency of passive sentences is far higher than in Chinese. Many passive sentences in English do not emphasize the implementer of action, or are unwilling to express the action implementer and are might as well omitted. For example, 长期以来大家都知道吸烟和肺癌 的关系. English expressed as: It has been known for a long time that there is some relationship between smoking and lung cancer.. 
The thinking of the Chinese is focused on the issuer of the action, while the British and Americans concern the carrier more. For example, 我希望你准是到那里. English expressed as: You are expected to be there on time..

\section{Different work orders: the attributes and adverbials in Chinese are often preposed, while those in English are often post-positioned}

The positions of attributes and adverbials involve in the word orders. Word order means the arrangement order of sentence components. The sentence components generally have a relatively fixed position, which cannot be changed at will generally. If changed, either the sentence does not make sense, or the meaning is changed, or the basic meaning is not changed, but the key point and effect of expression will be different. The order of Chinese sentences is generally spread in thinking order, while the thinking mode of the Chinese is to consider the environment and surrounding factors of the things first, and then the specific things and central event. In sentence pattern, the adverbials are often placed before the predicates or the main body of sentence, and the attributes, long or short, should be placed before the central word. (Wang Zhikui, 1997). By contrast, most of the English attribute components, except the words, are placed after the central word. The normal position of English adverbials is also behind the subject and predicate. English emphasizes the end weight, and any longer and words and cumbersome components are moved to the end of the sentence (Wang Zhikui, 1997). For example，是一个阅历丰富受人尊敬的人. English expressed as: He is a man rich in experience and respected by others. Another example, 他们过河的企图(pre-posted), their attempt to cross the river (post-positioned). In the English complex sentences, the subordinate clause expressing the time can be placed wither behind or before the main sentence, while in Chinese, the things first occurring are described first, and those occurring later and described later. For example, 我吃了晚饭后出去散步. I went out for a walk after I had my dinner. (Subordinated clause is behind the main sentence).

\section{Conclusion}

Through the analysis on the syntax difference between English and Chinese, we can see that the thinking mode of the Chinese influences the expression mode of Chinese, while the Chinese expression mode disturbs the students' expression in English. Aimed at this situation, we can collect more relevant models and data, and make appropriate Chinese and English comparative analysis in teaching to make students overcome the negative differences of the Chinese on English learning.

\section{References}

[1] Li Boli, Phenomenon of Negative Language Transfer and Generation of Chinglish [J], Journal of Chongqing Technology and Business University, 2005, (2).

[2] Zhou Li, Peng Jinding, Analysis on Negative Transfer of Native Language Syntax in English Composition of Chinese College Students [J], Journal of Hunan Institute of Technology, 2006 (10)

[3] Dai Weidong, Wang Dong, Research on Language Transfer: Problem and Thinking [J], Foreign Language, 2002 (6)

[4] Liu Lanying, Wu Jiazhen, Chinese Expression (M), Nanning: Guangxi Education Press, 2001 